AperTO - Archivio Istituzionale Open Access dell'Università di Torino

\title{
Allocentric and contra-aligned spatial representations of a town environment in blind people
}

\section{This is the author's manuscript}

Original Citation:

Availability:

This version is available http://hdl.handle.net/2318/1647041

since 2018-03-19T10:02:16Z

Published version:

DOI:10.1016/j.actpsy.2017.08.001

Terms of use:

Open Access

Anyone can freely access the full text of works made available as "Open Access". Works made available under a Creative Commons license can be used according to the terms and conditions of said license. Use of all other works requires consent of the right holder (author or publisher) if not exempted from copyright protection by the applicable law. 


\section{(3) \\ UNIVERSITÀ DEGLI STUDI DI TORINO}

This is an author version of the contribution published on:

Questa è la versione dell'autore dell'opera:

Acta Psychologica, 180, 2017, DOI: 10.1016/j.actpsy.2017.08.001.

The definitive version is available at:

La versione definitiva è disponibile alla URL:

http://www.sciencedirect.com/science/article/pii/S0001691817300549 
Allocentric and contra-aligned spatial representations of a town environment in blind people

Silvia Chiesa, Susanna Schmidt, Carla Tinti and Cesare Cornoldi

Silvia Chiesa

silvia.chiesa@unito.it

+390116702979

University of Turin, via Verdi 10.10124 Turin.

Corresponding author: Susanna Schmidt

susanne.schmidt@unito.it

+390116702874

University of Turin, via Verdi 10.10124 Turin.

Carla Tinti

carla.tinti@unito.it

+390116702896

University of Turin, via Verdi 10. 10124 Turin.

Cesare Cornoldi

cesare.cornoldi@unipd.it

+390498276603

University of Padova, via Venezia 8. 35131 Padova. 


\section{Allocentric and contra-aligned spatial representations of a town environment in blind people.}

Abstract

Evidence concerning the representation of space by blind individuals is still unclear, as sometimes blind people behave like sighted people do, while other times they present difficulties. A better understanding of blind people's difficulties, especially with reference to the strategies used to form the representation of the environment, may help to enhance knowledge of the consequences of the absence of vision. The present study examined the representation of the locations of landmarks of a real town by using pointing tasks that entailed either allocentric points of reference with mental rotations of different degrees, or contra-aligned representations.

Results showed that, in general, people met difficulties when they had to point from a different perspective to aligned landmarks or from the original perspective to contra-aligned landmarks, but this difficulty was particularly evident for the blind. The examination of the strategies adopted to perform the tasks showed that only a small group of blind participants used a survey strategy and that this group had a better performance with respect to people who adopted route or verbal strategies. Implications for the comprehension of the consequences on spatial cognition of the absence of visual experience are discussed, focusing in particular on conceivable interventions.

Highlights:

- Blind and blindfolded sighted participants explored a tridimensional city map

- They performed pointing tasks entailing allocentric and contra-aligned representations

- Performance decreased with increasing mental rotations and contra-aligned representations

- Differences between blind and sighted people were observed

- Participants who used a survey strategy performed better Keywords:

Spatial representation; blindness; egocentric/allocentric representation; contra-aligned representation; alignment effect; strategies. 


\section{Introduction}

The generation of spatial representations of environment is a daily and very important human activity, which enables to move efficiently in large- and small-scale environments (Hegarty, Montello, Richardson, Ishikawa, \& Lovelace, 2006). Representations of space, for example of a city, are important to reach different locations, to memorize the localization of different places, and to know the spatial relationships between them.

In this framework, some studies focusing on spatial representations used by people to move in a locomotion's space, distinguished between route and survey representations. In particular, route knowledge of environment is based on a serial representation of subsequent landmarks, while a survey representation entails an aerial or map-like view, also including information not obtainable from the direct experience with the environment (Brunyé \& Taylor, 2008; Taylor \& Tversky, 1992). The use of one rather than the other of these types of representations depends on the preferred cognitive style of the person or on external factors (e.g., survey representation is necessary when there are obstacles on the usual way that require a deviation). The route representation, in particular when moving in the space, uses the subject's body as reference and is egocentric, while the survey representation can be independent from the observer and, in this case, it can be allocentric. More in detail, allocentric representation permits an objects-to-objects type of representation, independently from the subject's position (Ekstrom, Arnold, \& Iaria, 2014). Therefore, an allocentric representation can refer also to the mental image created from a different viewpoint with respect to the own real position. For instance, according to Sholl's model (2001), to point at object B from the position of object A, people use the allocentric system to translate the represented self in the position of object A, and to re-represent the correct location of object B (Avraamides \& Kelly, 2008). 
Several studies investigated these cognitive abilities (e.g., Denis \& Loomis, 2007; Proulx, Todorov, Aiken, \& de Sousa, 2016), and some of them also focused on the role of vision by comparing sighted and blind participants (for a review see Cattaneo et al., 2008, and Pasqualotto \& Proulx, 2012). In general, results showed that blind people prefer route-like representations, while sighted individuals have the tendency to code spatial information in form of externally, surveybased representations (Millar, 1994; Noordzij, Zuidhoek, \& Postma, 2006), although sometimes blind people may be successful also in survey-representation tasks (Tinti, Adenzato, Tamietto, \& Cornoldi, 2006).

The blind people’s preference to use route representations could be linked to their tendency to rely on an egocentric frame of reference (Latini Corazzini, Tinti, Schmidt, Mirandola, \& Cornoldi, 2010; Thinus-Blanc \& Gaunet, 1997), maybe also due to the few opportunities offered to them to create survey representations. Blind people's spatial exploration is indeed mainly based on serial information, like auditory, tactile, motor and kinesthetic information (Postma, Zuidhoek, Noordzij, \& Kappers, 2008). Moreover, the guidance offered to them, when accompanied by sighted people, is typically route based. This sequential exploration of space seems to have a direct link with the egocentric frame of reference (Ruotolo, Ruggiero, Vinciguerra, \& Iachini, 2012). On the contrary, topographical knowledge of space, such as the survey one, is global and synchronous and generally implies an allocentric frame of reference, which is hardly accessible by a sequential exploration of space. Therefore, mainly relying on body-centered coding systems, blind individuals may have difficulties with processing allocentric spatial relations (Gaunet \& Rossetti, 2006; Ruggiero, Ruotolo, \& Iachini, 2009; Coluccia, Mammarella, \& Cornoldi, 2009; Iachini, Ruggiero, \& Ruotolo, 2014).

Indeed, in a study by Pasqualotto, Spiller, Jansari and Proulx (2013) involving a pointing task, late blind and sighted participants showed better performance in the allocentric condition, while congenitally blind persons showed better results in the egocentric condition, suggesting that 
visual experience may be a requisite for the generation and the use of allocentric representations. However, there are studies which suggest that also blind individuals may solve tasks which require the adoption of an allocentric perspective (Ittyerah, Gaunet, \& Rossetti, 2007; Eardley, Edwards, Malouin, \& Kennedy, 2015; Ruggiero, Ruotolo, \& Iachini, 2012). These contrasting results could be explained considering the different factors which may influence blind people’s performance, such as their mobility skills (Fiehler, Reuschel, \& Rösler, 2009; Schmidt, Tinti, Fantino, Mammarella, \& Cornoldi, 2013), the strategies they use to perform the tasks (Cornoldi, Tinti, Mammarella, Re, \& Varotto, 2009; Szubielska, 2014), or the difficulty of the tasks (Kozhevnikov \& Hegarty, 2001).

The allocentric representation assumes high relevance especially in situations in which people have to move in locomotion spaces such as a city and are required to go beyond the routinary pathways. Moving in the streets, we change our position with respect to the one held at the moment of encoding; the localization of the buildings with respect to ourselves continuously changes, and sometimes specific landmarks shift behind our own body. In these cases, the importance of a global and allocentric spatial representation, essential to apprehend the space from different points of view decentralized from the egocentric one, appears evident. However blind people only rarely are offered the opportunity of developing this type of representation and the learning material typically used is relatively abstract (e.g., Iachini et al., 2014; Schmidt et al., 2013).

Taking into account both the importance of an allocentric representation for moving across towns and the partly contradictory results of previous research, a first aim of the present work, associated with the administration of a first task, was to explore the capacity of blind and blindfolded sighted people to form allocentric spatial representations of a city starting from the exploration of a 3D tactile map. In particular, the participants’ abilities to understand the relationships between different landmarks and to form representations of the space from different points in the environment were explored, by asking them to imagine how the environment appears 
from different locations (e.g., "Imagine that you are in A, point to B”) (Hegarty \& Waller, 2004). Since the contrasting results found in previous studies could be related to the difficulty of the task (Kozhevnikov \& Hegarty, 2001), more than to a general incapacity to form allocentric representations, in the present study the degree of rotation necessary to perform the task was varied $\left(0^{\circ}, 90^{\circ}, 180^{\circ}\right)$. We expected to find a decrease in the performance with the increase of the degrees of the mental rotation required to perform the task in both groups (Wraga, Creem, \& Proffitt, 2000), but we intended to examine whether the difficulty was greater in the case of blind people also considering their reduced opportunity of developing survey representations of the environment.

However, a difficulty in the representation of the locations of a town can be present even if the person can maintain the position. In fact, moving in a city not only requires the construction of spatial representations from viewpoints different with respect to the egocentric one, but also the representation of the environment as it appears behind the person. In this case, the necessary representation is egocentric but contra-aligned (also called 'rotation condition', Rieser, 1989), i.e., a representation of a not directly perceivable space, involving the construction of correct spatial relations between landmarks opposed with respect to the orientation typically assumed, is required. Therefore, a second aim of the present study, associated with a second task, was to investigate how people represent space as it appears behind the own body. Results present in literature suggest that generally the representation aligned with the own body is more accurate than the contra-aligned representation (Borella, Meneghetti, Muffato, \& De Beni, 2015; Cerles, Gomez, \& Rousset, 2015; Pazzaglia \& De Beni, 2006), defining the so-called “alignment effect” (Levine, Jankovic, \& Palij, 1982; Kelly, Avraamides, \& Loomis, 2007). Apparently, the contra-aligned task should entail a similar difficulty for both sighted and blind participants as the egocentric frame of reference can be maintained. However, it has been demonstrated that the success in the contra-aligned tasks is related with the ability to generate survey representations (Borella et al., 2015), and therefore it is possible that also in this case blind individuals meet more difficulties than the sighted ones. Just a few 
studies investigated blind people's ability to represent the space behind their body and results are rather contradictory. In particular, in a study in which blind and sighted participants were asked to relocate objects on a table, the two groups showed a good and comparable performance in the aligned condition, while blind participants performed worse than the sighted ones in the contraaligned condition (Coluccia et al., 2009). On the contrary, in a study by Giudice, Betty and Loomis (2011), blind and sighted participants showed similar performances not only in the aligned but also in the contra-aligned condition after the haptic learning of a map.

In sum, given the variability of results and the methodologies used in different studies, there is need of further evidence concerning the ability of people, and especially of people without vision, to represent the locations of landmarks from a perspective different from the assumed one. Therefore, in this study we further investigated the ability of blind and blindfolded sighted participants to form spatial representations either from a different perspective or in a contra-aligned condition, using two pointing tasks.

In addition, considering previous results about the influence of the strategy used to achieve the task (Schmidt et al., 2013), and the implications of the cognitive style observed for the alignment effect (Nori \& Giusberti, 2003; Nori, Grandicelli, \& Giusberti, 2006), for both tasks we tested the possible influence of the strategies used by distinguishing two spatial types of strategies (construction of a survey representation, or a route representation), and a verbal strategy based on the memorization of a verbal description of the environment. We expected a better performance in participants using a survey strategy than in the ones using a route or a verbal strategy and we examined whether a predicted poorer performance of the blind in the tasks was associated to a minor use of the survey strategy as suggested by Schmidt et al. (2013).

For the construction of a spatial representation of a town, in order to make the testing situation comfortable, concrete and plausible for the participants, we took advantage of the availability of a tridimensional realistic map representing the city center of the town of Turin (96 
cm x $132 \mathrm{~cm}$, 1:1000 scale), capital of the region where the participants live. The map represents buildings of the city, streets and squares in a three-dimensional way, so as to give the explorer a complete and realistic idea of the city center.

We adopted a method that involved the memorization of the location of a series of landmarks based on the tactile exploration of the town through a given pathway going along with a description of these landmarks. To control for an eventual influence of previous experience with the town, we included the description of both existing landmarks (hereinafter 'known landmarks') and unknown buildings/squares (hereinafter 'unknown landmarks') we rendered landmarks by highlighting a tactile cue during the exploration (for an example, see the procedure section). We then administered a pointing task which required to indicate a specific landmark imagining to be at another landmark met during the pathway, and then a second pointing task that requested the adoption of the initial position and asked for the pointing of different landmarks first in the familiar aligned perspective and then from a contra-aligned perspective. Finally, participants were invited to specify the strategy they had adopted for representing the town and doing the tasks.

\section{Method}

\subsection{Participants}

The study involved 38 participants, divided in two groups. The first group was composed by 19 early totally blind people (mean age 47.2; $S D=12.4$, range from 27 to 68,11 males) recruited through the local blind institution (UICI - Unione Italiana Ciechi ed Ipovedenti). We considered people to be totally blind when they were not able to perceive shapes and positions of the objects, and early blind when the visual deficit was present at birth or appeared in the first three years of life (Thinus-Blanc \& Gaunet, 1997). We included in the sample all congenitally blind adults who were 
willing to participate in the study with the only exclusion criterion that they should not present other sensory, physical or cognitive deficits. Based on this criterion, no voluntary participant has been excluded. We also tested a group of 19 blindfolded sighted participants matched for gender (11 males), age $(M=47.3$; $S D=12.1$; range from 30 to 64$)$, schooling $(n=4$ : junior high school, $n=7$ : high school, $n=8$ : university degree) and familiarity with the center of Turin. All participants lived in the region Piedmont where Turin is located.

\subsection{Procedure and materials}

Before starting the two experimental tasks, the participants were interviewed about their knowledge of the city of Turin. In more detail, we examined their knowledge of 15 famous buildings or squares of the city center, and we found that blind and sighted participants presented a similar knowledge of the city of at least 10 out of 15 of these famous buildings or squares.

The two experimental tasks were based on the use of a tridimensional realistic map representing the city center of the town (96 cm x $132 \mathrm{~cm}, 1: 1000$ scale) created by Protocube Reply for the UICI of Turin (Figure 1). The map represents buildings of the city, streets and squares in a three-dimensional way, so as to give the explorer the impression of really moving through the city.

After the sequential exploration of the tridimensional map (learning phase), participants were administered two tasks. In the first task (allocentric) they had to imagine to move from one landmark to another, in such a way that the landmarks resulted equally or differently oriented with respect to the participant's position, and to point to other landmarks. Successively, in the second task (contra-aligned), participants were first invited to point to the landmarks from their own position with the map in front of them, and then asked to do the same in a contra-aligned way. Finally, participants were invited to specify the strategy they had used to memorize the itinerary and the localization of the landmarks and doing the tasks. 
All participants were tested individually and sighted participants were blindfolded before entering the experimental room.

During the learning phase, each participant was positioned in front of the map and the experimenter guided his/her dominant hand through a specific learning itinerary and invited the participant to haptically explore a predetermined set of landmarks. The route of the exploration is represented in Figure 1. The starting point of the exploration was the center of Piazza San Carlo. During the exploration, the experimenter described the streets and the buildings that participants explored and asked them to memorize the localization of 12 specific landmarks. In more detail, the experimenter informed the participant about the name of the street where he/she found him/herself in a given moment of the exploration. Moreover, the experimenter told the names of the landmarks and focalized the participant's attention on some of their specific details. For example, in front of “Mr. Bianchi’s house”, the experimenter, guiding the participant’s hand, said “As you can feel, at the beginning of this street there are some buildings and the third one has a courtyard. I ask you to memorize the localization of this house. Here leaves Mr. Bianchi who usually gets out with his dog at around 5 pm to make a walk.”

To control for the influence of previous knowledge of the city, the itinerary was characterized by six known landmarks of the city and six unknown landmarks marked by us, e.g., Mr. Bianchi’s house with a particular courtyard (variable ‘Known’). The differentiation of the landmarks was based on judgements of a group of experts knowing the background of the participants and individuating six landmarks whose existence was known to all participants, as further confirmed by the interview assessing their knowledge of the city. The exploration was repeated twice to guarantee a good memorization of the landmarks' localizations. The learning phase lasted about 15 minutes.

--- Insert Figure 1 about here --- 
After the exploration of the map, participants were engaged in two pointing tasks where they had to indicate the localization of different landmarks by using a mechanical pointer similar to a crane. This crane was featured by a rotating arm that participants had to move to indicate their responses and by a pointer used to mark their answers on a tally sheet.

In the first task, the questions aimed at investigating allocentric spatial representations. Participants had to imagine to be outside a specific known or unknown building and to point a second known or unknown building from the imagined perspective. For instance, we asked participants "to point $B$, as if facing $A$ ". The task required to imagine different body orientations, without actually moving the body. In more detail, participants had to adopt different perspectives, performing a $0^{\circ}, 90^{\circ}$ or $180^{\circ}$ mental rotation. We proposed 9 trials in which the variable Known and the rotation $\left(0^{\circ}, 90^{\circ}, 180^{\circ}\right)$ were systematically varied. In 3 trials $\left(0^{\circ}, 90^{\circ}, 180^{\circ}\right)$ both buildings were known, in 3 trials $\left(0^{\circ}, 90^{\circ}, 180^{\circ}\right)$ building A was known and building B unknown, in 3 trials $\left(0^{\circ}, 90^{\circ}, 180^{\circ}\right)$ both buildings were unknown. Figures 2 to 4 represent the position of the participant and the mental rotations required to solve the task in the three conditions of rotation. The head depicted inside the map represents the imagined perspective adopted to answer to the questions. In front of the map, the participant's position, the pointer and the tally sheet for the responses.

--- Insert Figure 2 about here ---

--- Insert Figure 3 about here ---

--- Insert Figure 4 about here --- 
Subsequently, we investigated the aligned and the contra-aligned representations. In this second task, participants first had to imagine to have the map in front of them (aligned condition), and to indicate with the use of the pointer the localization of 6 landmarks, half known and half unknown. Afterwards, they were asked to imagine having the map behind of them and to indicate the localization of 6 other landmarks, half known and half unknown. In this condition, the tally sheet was rotated by $180^{\circ}$. Figures 5 and 6 represent the position of the participant with respect to the location of the imagined map in the aligned and contra-aligned conditions.

--- Insert Figure 5 about here ---

--- Insert Figure 6 about here ---

At the end of these two tasks, we interviewed the participants about the strategies they had used to complete them. In more detail, we investigated whether during the exploration, participants created a sequential representation based on the itinerary in the map (route strategy), or a global representation, as they looked over the map (survey strategy), or whether they memorized the verbally described directions (verbal strategy). The participants were explicitly invited to choose one among these alternatives describing the different strategies. In the case they were not able to decide for one among these three strategies, their answers were categorized as 'other' and recoded in a second time by two experts.

\section{Results}

Data analyses were computed by using SPSS, version 23. For both tasks and for each trial, we first calculated the angular error between the point indicated by the participant and the correct position of the landmark. Since the landmarks were located in different points in the map, the 
maximum degree of error differed for each landmark. Thus, the degree of error was adjusted by the maximum error degree possible for a specific landmark (range from $0=$ no error, to $1=$ maximum error). An arcsine transformation was computed on these adjusted degrees of error for all analyses. Finally, for each of the two tasks and the different conditions we controlled whether there were outliers. Responses that deviated more than a Z-score equal to 2.68 from the mean of the whole sample in the different conditions were considered as outliers. By adopting this criterion no outlier responses were observed.

First the results obtained by the participants in the two tasks will be presented. Afterwards, the strategies used to solve the task will be described and the two pointing tasks will be reanalyzed by taking into account also the strategies used to perform them.

As to the allocentric task, we first tested whether the variable Known had an influence on the performance by computing a GLM (General Linear Model) repeated measures analysis on the arcsine-transformed error degrees. Results showed that the variable Known had no significant effect, $F(2,36)=.15, p=.86$, indicating that previous knowledge of the landmarks did not affect the results; thus, this variable was not considered for further analyses.

We then calculated the mean pointing error for the three items that required a mental rotation of $0^{\circ}, 90^{\circ}$, and $180^{\circ}$ respectively. Table 1 shows the means of the adjusted degrees of error for the two groups in the different conditions of mental rotation. Overall, pointing errors varied from .22 to .45 . In the $0^{\circ}$ condition the performance was better in both groups with respect to the two other conditions, and blind participants performed worse than the sighted ones in all three conditions. 
To assess whether the differences observed at a descriptive level were significant, a GLM repeated measures analysis was computed on the arcsine-transformed error degrees by considering Group (Blind vs Sighted) as between subject variable, and Condition $\left(0^{\circ}, 90^{\circ}, 180^{\circ}\right)$ as within subject variable. Results showed a main effect of degrees of mental rotation required to solve the task, $F(2,35)=7.22, p<.01, \eta^{2} p=.29, \beta=.91$. Pair-wise comparisons revealed that both groups did better in the $0^{\circ}$ condition $(E M M=1.00, E S E=.05)$ than in the $90^{\circ}(E M M=1.24, E S E=.05$; $\left.F(1,36)=11.59, p<.01, \eta^{2} p=.24, \beta=.91\right)$ and in the $180^{\circ}$ condition $(E M M=1.24, E S E=.08$; $\left.F(1,36)=11.13, p<.01, \eta_{p}^{2}=.24, \beta=.90\right)$, the two latter not differing significantly.

In addition, there was a significant effect of group, $F(1,36)=7.54, p<.01, \eta^{2} p=.17, \beta=.76$, as the performance was better for sighted participants $(E M M=1.04, E S E=.06)$ than for the blind ones $(E M M=1.29, E S E=.06)$. The interaction between condition and group only approached significance, $F(2,35)=2.92, p=.07$, but it can be seen in Table 1 that the group difference was particularly great in the case of the $180^{\circ}$ rotation.

Subsequently, we analyzed the performance of the participants in the aligned and contraaligned task. After the investigation of the representation of the space as it appears from different points in the city, this task should allow to understand the characteristics of space representation from the own point of view and to understand how people represent objects located behind the body.

As for the task about the allocentric representations, we first tested whether the variable Known had an influence on the performance by computing a GLM repeated measures analysis on arcsine-transformed error degrees. Results revealed that also in this case the variable Known had no significant effect, $F(1,37)=.70, p=.41$, thus it was not considered for further analyses.

We then calculated the mean pointing error for the 6 aligned and the 6 contra-aligned trials. Table 2 shows the means of the adjusted degrees of error for the two groups in the two conditions. As we can see, overall the performance seemed to be better with respect to the previous task, mean 
error degrees ranging from .13 to .27. Moreover, sighted participants showed lower degrees of error than blind participants, and the contra-aligned condition was more difficult than the aligned condition for both groups, but especially for the blind one.

\section{--- Insert Table 2 about here ---}

A GLM repeated measures analysis was computed on the arcsine-transformed error degrees by considering Group (Blind vs Sighted) as between subject variable, and Alignment (Aligned vs Contra-aligned) as within subject variable. Results showed that both groups were more accurate in the aligned $(E M M=.77, E S E=.03)$ than in the contra-aligned condition $(E M M=.95, E S E=.05$; $\left.F(1,36)=19.61, p<.001, \eta^{2} p=.35, \beta=.99\right)$. There was also a main effect of group, $F(1,36)=6.03$, $p<.05, \eta^{2} p=.14, \beta=.67$, as sighted participants generally performed better $(E M M=.78, E S E=$ $.05)$ than blind participants $(E M M=.94, E S E=.05)$. The interaction between group and condition only approached significance, $F(1,36)=3.01, p=.09$, but it is evident from Table 2 that the blinds' difficulty was mainly associated with the contra-aligned condition.

As regards the strategies used to solve the tasks, all participants reported to have used a preferred strategy consistently across trials and no difficulties were found by two experts to reassign participants who chose the 'other' strategy $(n=4)$ to one of the three strategies we had decided to consider, as the agreement between them was complete. Table 3 reports the strategies used by the two groups of participants. Frequencies show the same preference for both blind and sighted participants in the use of the verbal strategy, while a chi-square analysis showed a significant different tendency in the use of survey and route strategies in the two groups $\left(X^{2}(1, N=30)=5, p<\right.$ .05). Sighted participants tended to use more frequently the survey strategy, whereas blind participants mostly used the route strategy. 
Despite the fact that some cells had a limited number of observations, we considered it important to explore the impact of strategies on participants’ performances and on eventual group differences. Concerning the allocentric task, we again calculated the means of the adjusted degrees of error for the two groups by considering the different conditions of mental rotation and the strategies used to perform the task (Table 4). Generally, participants had a good performance in the $0^{\circ}$ condition, independently from the strategy used. In the $90^{\circ}$ and $180^{\circ}$ conditions the performance decreased, in particular in participants who used the verbal strategy. Moreover, the blind participants who used the route strategy in the $180^{\circ}$ condition had also a rather poor performance.

\section{--- Insert Table 4 about here ---}

A GLM repeated measures analysis was computed on the arcsine-transformed error degrees by considering Group (Blind vs Sighted) and Strategy (Verbal, Survey, Route) as between subject variables, and Condition $\left(0^{\circ}, 90^{\circ}, 180^{\circ}\right)$ as within subject variable. Results showed again a main effect of degrees of mental rotation required to solve the task, $F(2,31)=7.88, p<.01, \eta^{2}{ }^{2}=.34, \beta=$ .93 , the $0^{\circ}$ condition yielding significantly lower errors $(E M M=.99, E S E=.06)$ than the $90^{\circ}$ condition $\left(E M M=1.26\right.$, ESE $\left.=.06 ; F(1,32)=13.06, p<.01, \eta^{2} p=.29, \beta=.94\right)$ as well as the $180^{\circ}$ condition $\left(E M M=1.22, E S E=.08 ; F(1,32)=10.02, p<.01, \eta^{2}{ }_{p}=.24, \beta=.874\right)$, the two latter not differing significantly.

In addition, there was a significant interaction effect of condition by strategy, $F(4,64)=$ 2.68, $p<.05, \eta_{p}^{2}=.14, \beta=.71$ : while in the $0^{\circ}$ condition there was no difference in performance according to the strategy used, in the $90^{\circ}$ condition, performance was better for participants who 
used a survey strategy $(E M M=1.11, E S E=.10)$ than for the ones who used a verbal strategy $(E M M$ $=1.42, E S E=.11, t(32)=2.04, p<.05)$, and in the $180^{\circ}$ condition, participants who used a survey strategy $(E M M=.93, E S E=.15)$ did better than both the participants who used a verbal $(E M M=$ $1.40, E S E=.16, t(32)=2.20, p<.05)$ or a route strategy $(E M M=1.33, E S E=.11, t(32)=2.17, p<$ .05). No other significant main or interaction effects were observed, in particular, the effect of group was no longer significant, suggesting that strategies played an important role in the task performance and their impact absorbed the differences between blind and sighted participants. It must be noticed that not all participants within each subgroup presented the same pattern of performance. For example, two blind participants within the survey strategy subgroup took advantage from this strategy in the most crucial $180^{\circ}$ rotation condition, whereas the third participant adopting this strategy had an error close to the mean error observed for the whole group of blind. On the contrary, giving a closer look to the blind participants in the verbal subgroup in the same condition, three out of four participants behaved poorly, but one had a good performance.

Finally, as to the influence of strategies used on the performance of participants in the aligned and contra-aligned task, we again calculated the means of the adjusted degrees of error for the two groups in the two conditions and by considering also the strategies used to perform the task (Table 5).

In general, participants had a quite good performance in the aligned condition, with the exception of the blind people who used a verbal strategy. In the contra-aligned condition, the performance of participants was worse. In particular, blind people who used the verbal strategy had a mean error degree near to .50 .

--- Insert Table 5 about here --- 
A GLM repeated measures analysis computed on the arcsine-transformed error degrees by considering Group (Blind vs Sighted) and Strategy (Verbal, Survey, Route) as between subject variables, and Condition (Aligned vs Contra-aligned) as within subject variable confirmed that both groups were more accurate in the aligned $(E M M=.77, E S E=.04)$ than in the contra-aligned condition $\left(E M M=1.00, E S E=.04 ; F(1,32)=39.97, p<.001, \eta^{2} p=.49, \beta=1.00\right)$, and that sighted $(E M M=.79, E S E=.04)$ did better than blind participants $(E M M=.97, E S E=.05 ; F(1,32)=7.43, p$ $\left.<.05, \eta^{2}{ }^{2}=.19, \beta=.75\right)$. Moreover, the interaction group by alignment reached significance, $F(1,32)=6.15, p<.05, \eta^{2} p=.16, \beta=.67$; blind participants $(E M M=1.14, E S E=.06)$ found more difficulty with the contra-aligned condition than the sighted ones $(E M M=.85, E S E=.06 ; F(1,32)=$ 11.20, $p<.01, \eta^{2}{ }^{2}=.26, \beta=.90$ ), while in the aligned condition no significant difference between the two groups was observed.

Again, we also found a main effect of strategy, $F(2,32)=5.46, p<.01, \eta^{2} p=.25, \beta=.81$. Pair-wise comparisons revealed that the verbal strategy $(E M M=1.04, E S E=.07)$ led to worse results than both the survey $(E M M=.76, E S E=.06, t(32)=3.19, p<.01)$ and the route strategy $(E M M=.84, E S E=.05, t(32)=2.58, p<.05)$, the two latter not differing from each other. In addition, there was an interaction alignment by strategy, $F(2,32)=3.79, p<.05, \eta^{2}{ }_{p}=.19, \beta=.65$ : participants using the verbal strategy performed worse in the contra-aligned $(E M M=1.24, E S E=$ $.08)$ than in the aligned condition $(E M M=.85, E S E=.07, t(32)=4.97, p<.001)$; similarly, participants using the route strategy performed worse in the contra-aligned $(E M M=.91, E S E=.06)$ than in the aligned condition $(E M M=.77, E S E=.05, t(32)=2.55, p<.05)$; on the contrary, participants using a survey strategy showed no significant difference in the performance between the aligned $(E M M=.70, E S E=.07)$ and the contra-aligned condition $(E M M=.83, E S E=.08)$.

Also in this case we considered the individual performances within each strategy subgroup with reference to the crucial contra-aligned condition and we found that two out of the three blind participants who used the survey strategy showed a very good performance, while the third showed 
a degree of error corresponding to the mean degree of error found in the overall group of blind participants. In the case of the use of the verbal strategy, three out of four blind participants had an error higher than the other blinds, thus confirming the low efficacy of this strategy.

\section{Discussion}

In this study, we investigated the ability of blind and blindfolded sighted people to construct spatial representations of a city on the basis of a realistic 3D model and its tactile exploration along a pathway. In particular, we examined the participants' ability to represent the locations of different landmarks adopting a perspective different from the one assumed during the city exploration, either allocentric, or contra-aligned. Specifically, participants carried out two pointing tasks: the first involved allocentric points of reference and mental rotations of different degrees, the second investigated aligned and contra-aligned representations.

By using a realistic representation of a city combined with the instruction to imagine to move across it, the study had to offer the participants the impression of exploring a real and meaningful environment. In fact, participants reacted positively to the task and reported the sensation as if they were really moving in the city.

Results showed that overall blind people tended to perform worse in both tasks than the sighted ones. This result appears in line with previous studies that showed differences between blind and sighted people in spatial tasks (Cattaneo et al., 2008; Ruggiero et al., 2009; Ruggiero et al., 2012), and suggests that the condition of blindness could impair the ability of treating spatial information.

Concerning more specifically the allocentric task, although we observed a main effect of degrees for both groups, showing more errors with the increased angular degree of the imagined perspective, the difficulty with the $180^{\circ}$ degrees rotated position is particularly evident in the blind, 
further supporting the observation of blind people’s difficulty with rotated patterns (Postma, Zuidhoek, Noordzij, \& Kappers, 2007).

As to the second task, comparing the performance in the aligned and contra-aligned conditions, we found that the former was easier for both groups and also in this case a difference between blind and sighted participants emerged, as in both conditions sighted participants performed better than the blind ones. The difficulty in the contra-aligned condition is in line with previous evidences about the "alignment effect” (Levine et al., 1982). In fact, when people have to imagine the space as it appears behind their body, their performance decreases. This effect studied in sighted people was here extended to blind people, who actually experienced a particular difficulty in the contra-aligned condition. As this second task is substantially different from the first one (both as regards the reference frame and the operations required to the participants), this result suggests that the condition of blindness could affect the processing of spatial images across tasks (Millar, 1994; Morrongiello, Timney, Humphrey, Anderson, \& Skory, 1995; Pasqualotto \& Newell, 2007; Pasqualotto et al., 2013).

Behind these general results about group differences, the present study also aimed to evaluate the eventual effect of the strategies used to perform the tasks. Research on the effects of total early blindness on spatial representation necessarily tests small groups and, also for this reason, normally does not deeply examine the influence of the strategies adopted by the participants, losing information that may be very important (Cornoldi et al., 2009; Schmidt et al., 2013). Therefore, despite the fact that the sample size was relatively small also in the present study, we explored the effects of the strategies finding that they played an important role.

Firstly, the different disposition in the use of strategies in the two groups was evident. Generally, the survey strategy was mainly adopted by sighted participants and the route strategy by blind people (Millar, 1994; Noordzij et al., 2006). Moreover, the survey strategy permitted to perform with lower degrees of error than the route and verbal strategies. In particular, the survey 
strategy helped participants to solve with lesser degrees of error the $180^{\circ}$ condition of the allocentric task, and both the aligned and contra-aligned conditions. In addition, participants who used the survey strategy performed better than people who used the verbal strategy in the $90^{\circ}$ condition. These results confirm that the survey strategy is advantageous in the manipulation of spatial representations (Meneghetti, De Beni, Pazzaglia, \& Gyselinck, 2011; Nori \& Giusberti, 2003; Pazzaglia \& Taylor, 2007). At the opposite, the verbal strategy was associated with the worst performance in both tasks.

It is interesting to observe that by taking into account the strategies used, the group difference in the first task disappeared, suggesting that, at least in this case, this difference was not due to blindness per se, but to the fact that blind people use to a lower extent the most appropriate survey strategy. On the contrary, the fact that, in the second task, a significant group difference remained even when strategies were taken into consideration, suggests that in this case (and especially in the contra-aligned condition) the adoption of the survey strategy is not sufficient for eliminating the consequences of the absence of vision. On this respect, it is interesting to observe that apparently the representation of contra-aligned landmarks seems to refer to elements that are not based on vision, as by their nature they are not visible. However, it is possible that the absence of prior visuospatial experience makes it difficult to implement the processes that support the elaboration of contra-aligned material (Borella et al., 2015).

The results of this study provide further evidence on spatial cognition in general and on blind people's spatial cognition in particular. Being forced to adopt a perspective different from the one adopted during encoding impairs performance and this applies also to the case of representing the directions of landmarks of a city explored in a micromodel format. However, the present study has also some limitations, the first regarding the rather small sample size. Although congenitally completely blind people constitute a very interesting experimental group to gather insight on the role of vision in spatial representation, it is rather difficult to recruit a consistent group of 
participants with these characteristics. This may have lowered the statistical power of some analyses especially the ones considering also the strategies used to perform the tasks. Other limitations are related to the method we decided to use. For example, we were able to make the tasks friendly and likeable to our participants, but we could not balance their order and it cannot be excluded that this could have partly affected some of the results. Future research should examine this aspect and also focus on the use of different instruments to encode spatial representations, for example comparing the performance of participants after the exploration of a tactile map and after the direct visit in the city. Moreover, other variables that may influence the spatial representation of a city, as for example the geometric structure of the environment or its complexity, could be considered (Ekstrom et al., 2014). Finally, future research should examine whether the better performance obtained by the blind who used the survey strategy, especially in the $180^{\circ}$ allocentric and the contra-aligned conditions, is due to the use of the strategy per se or to some particular underlying abilities that bring to its use.

To conclude, it should be pointed out that no difference in the localization of known and unknown landmarks emerged, showing that participants were able to memorize the localization of both types of landmarks and that the previous knowledge of the city didn't influence the performance. In fact, all participants were able to acquire a comparable spatial knowledge about known as well as completely new landmarks. This result confirms the possibility to create spatial representations through the exploration of tactile 3D maps as this instrument permits a good memorization of landmarks and is useful and likeable for blind people to learn the configuration of a city (Almeida, Martins, \& Lima, 2015; Harder \& Michel, 2002). For this reason, we suggest that 3D tactile maps could be used to increase autonomy and mobility skills in blind people. Rehabilitators should consider these evidences and instruments in programs for orientation and mobility training to enhance the spatial abilities of blind people (Fiehler et al., 2009). In addition, survey strategy appears a powerful strategy for reducing errors, and also interventions to develop 
the abilities in the use of the survey strategy could benefit the autonomy of blind people. Obviously we cannot know if the choice of a survey strategy depends on particular skills not available to all participants (an aspect that could be examined by assessing the effects of a specific training); at the same time we have the impression that the infrequent choice of a survey strategy by the blind participants could be due to the typical way they experience town environments. In fact, blind people normally know the town by a sequential route-like exploration in company of a sighted person, with a limited possibility to acquire the relationships between different reference points, while the exploration of a tactile map could favour the creation of a more global survey representation. 
References

Almeida, M. de F. X. M., Martins, L. B., \& Lima, F. J. (2015). Analysis of Wayfinding Strategies of Blind People Using Tactile Maps. Procedia Manufacturing, 3, 6020-6027. doi:10.1016/j.promfg.2015.07.716

Avraamides, M. N, \& Kelly, J. W. (2008). Multiple systems of spatial memory and action. Cognitive Processing, 9, 93-106. doi:10.1007/ s10339-007-0188-5

Borella, E., Meneghetti, C., Muffato, V. \& De Beni, R. (2015). Map learning and the alignment effect in young and older adults: how do they gain from having a map available while performing pointing tasks? Psychological Research, 79, 104-119. doi:10.1007/s00426-014-0543-y

Brunyé, T. T., \& Taylor, H. A. (2008). Working memory in developing and applying mental models from spatial descriptions. Journal Of Memory And Language, 58(3), 701-729. doi:10.1016/j.jml.2007.08.003

Cattaneo, Z., Vecchi, T., Cornoldi, C., Mammarella, I. C., Bonino, D., Ricciardi, E., \& Pietrini, P. (2008). Imagery and spatial processes in blindness and visual impairment. Neuroscience And Biobehavioral Reviews, 32(8), 1346-1360. doi:10.1016/j.neubiorev.2008.05.002

Cerles, M., Gomez, A., \& Rousset, S. (2015). An implicit spatial memory alignment effect. Cognitive Processing, 16(Suppl 1), 179-183. doi:10.1007/s10339-015-0694-9

Coluccia, E., Mammarella, I. C., \& Cornoldi, C. (2009). Centred egocentric, decentred egocentric, and allocentric spatial representations in the peripersonal space of congenital total blindness. Perception, 38(5), 679-693. doi:10.1068/p5942

Cornoldi, C., Tinti, C., Mammarella, I. C., Re, A. M., \& Varotto, D. (2009). Memory for an imagined pathway and strategy effects in sighted and in totally congenitally blind individuals, Acta Psychologica, 130, 11-16. doi:10.1016/j.actpsy.2008.09.012

Denis, M., \& Loomis, J. M. (2007). Perspectives on human spatial cognition: Memory, navigation, and environmental learning. Psychological Research, 71(3), 235-239. doi:10.1007/s004260060079x

Eardley, A. F., Edwards, G., Malouin, F., \& Kennedy, J. M. (2015). Allocentric Spatial Performance Higher in Early-Blind and Sighted Adults Than in Retinopathy-of-Prematurity Adults. Perception, 45(3), 281-99. doi:10.1177/0301006615607157 
Ekstrom, A. D., Arnold, A. E. G. F., \& Iaria, G. (2014). A critical review of the allocentric spatial representation and its neural underpinnings: toward a network-based perspective. Frontiers in Human Neuroscience, 8, 803. doi:10.3389/fnhum.2014.00803

Fiehler, K., Reuschel, J., \& Rösler, F. (2009). Early non-visual experience influences proprioceptive-spatial discrimination acuity in adulthood. Neuropsychologia, 47(3), 897-906. doi:10.1016/j.neuropsychologia.2008.12.023

Gaunet, F., \& Rossetti, Y. (2006). Effects of visual deprivation of space representation: immediate and delayed pointing towards memorised proprioceptive targets. Perception, 26, 107124.

Giudice, N. A., Betty, M. R., \& Loomis, J. M. (2011). Functional equivalence of spatial images from touch and vision: Evidence from spatial updating in blind and sighted individuals. Journal Of Experimental Psychology: Learning, Memory, And Cognition, 37(3), 621-634. doi:10.1037/a0022331

Harder, A., \& Michel, R. (2002). The target-route map: evaluating its usability for visually impaired persons. Journal of Visual Impairment \& Blindness (JVIB), 96(10), 711-723.

Hegarty, M., Montello, D. R., Richardson, A. E., Ishikawa, T., \& Lovelace, K. (2006). Spatial abilities at different scales: Individual differences in aptitude-test performance and spatiallayout learning. Intelligence, 34, 151-176. doi:10.1016/j.intell.2005.09.005

Hegarty, M., \& Waller, D. (2004). A dissociation between mental rotation and perspectivetaking spatial abilities. Intelligence. 32, 175-191. doi:10.1016/j.intell.2003.12.001

Iachini, T., Ruggiero, G., \& Ruotolo, F. (2014). Does blindness affect egocentric and allocentric frames of reference in small and large scale spaces? Behavioural Brain Research, 273, 73-81. doi:10.1016/j.bbr.2014.07.032

Ittyerah, M., Gaunet, F., \& Rossetti, Y. (2007). Pointing with the left and right hands in congenitally blind children. Brain and Cognition, 64, 170-183. doi:10.1016/j.bandc.2007.02.002

Kelly, J. W., Avraamides, M. N., \& Loomis, J. M. (2007). Sensorimotor alignment effects in learning and novel environments. Journal of Experimental Psychology: Learning, Memory, and Cognition, 33, 1092-1107. doi:10.1037/0278-7393.33.6.1092

Kozhevnikov, M, \& Hegarty, M. (2001). A dissociation between object manipulation spatial ability and spatial orientation ability. Memory \& Cognition, 29, 745-756. doi:10.3758/BF03200477 
Latini Corazzini, L., Tinti, C., Schmidt, S., Mirandola, C., \& Cornoldi, C. (2010). Developing spatial knowledge in the absence of vision: allocentric and egocentric representations generated by blind people when supported by auditory cues. Psychologica Belgica, 50(3\&4), 327334. doi:10.5334/pb-50-3-4-327

Levine, M., Jankovic, I. N., \& Palij, M. (1982). Principles of spatial problem solving. Journal Of Experimental Psychology: General, 111(2), 157-175. doi:10.1037/0096-3445.111.2.157

Meneghetti, C., De Beni, R., Pazzaglia, F., \& Gyselinck, V. (2011). The role of visuo-spatial abilities in recall of spatial descriptions: A mediation model. Learning and Individual Differences, 21(6), 719-723.

Millar, S. (1994). Understanding and Representing Space: Theory and Evidence from Studies with Blind and Sighted Children. Oxford: Clarendon Press.

Morrongiello, B. A., Timney, B., Humphrey, G. K., Anderson, S., \& Skory, C. (1995). Spatial knowledge in blind and sighted children. Journal of experimental child psychology, 59(2), 211-233. doi:10.1006/jecp.1995.1010

Noordzij, M. L., Zuidhoek, S., \& Postma, A. (2006). The influence of visual experience on the ability to form spatial mental models based on route and survey descriptions. Cognition, 100(2), 321-342. doi:10.1016/j.cognition.2005.05.006

Nori, R., \& Giusberti, F. (2003). Cognitive styles: errors in directional judgments. Perception, 32(3), 307-320. doi:10.1068/p3380

Nori, R., Grandicelli, S., \& Giusberti, F. (2006). Alignment effect: primary-secondary learning and cognitive styles. Perception, 35(9), 1233-1249. doi:10.1068/p5351

Pasqualotto, A., \& Newell, F. N. (2007). The role of visual experience on the representation and updating of novel haptic scenes. Brain and cognition, 65(2), 184-194.

Pasqualotto, A., \& Proulx, M. J. (2012). The role of visual experience for the neural basis of spatial cognition. Neuroscience And Biobehavioral Reviews, 36(4), 1179-1187. doi:10.1016/j.neubiorev.2012.01.008

Pasqualotto, A., Spiller, M. J., Jansari, A. S., \& Proulx, M. J. (2013). Visual experience facilitates allocentric spatial representation. Behavioural Brain Research, 236(1), 175-179. doi:10.1016/j.bbr.2012.08.042 
Pazzaglia, F., \& De Beni, R. (2006). Are people with high and low mental rotation abilities differently susceptible to the alignment effect? Perception, 35(3), 369-383. doi:10.1068/p5465

Pazzaglia, F., \& Taylor, H. A. (2007). Perspective, instruction, and cognitive style in spatial representation of a virtual environment. Spatial Cognition and Computation, 7(4), 349-364.

Postma, A., Zuidhoek, S., Noordzij, M. L., \& Kappers, A. L. (2008). Haptic orientation perception benefits from visual experience: Evidence from early-blind, late-blind, and sighted people. Perception \& Psychophysics, 70(7), 1197-1206. doi:10.3758/PP.70.7.1197

Postma, A., Zuidhoek, S., Noordzij, M. L., \& Kappers, A. M. (2007). Differences between early-blind, late-blind, and blindfolded-sighted people in haptic spatial-configuration learning and resulting memory traces. Perception, 36(8), 1253-1265. doi:10.1068/p5441

Proulx, M. J., Todorov, O. S., Aiken, A. T., \& de Sousa, A. A. (2016). Where am I? Who am I? The relation between spatial cognition, social cognition, and individual differences in the built environment. Frontiers In Psychology, 7, 64. doi:10.3389/fpsyg.2016.00064

Rieser, J. J. (1989). Access to knowledge of spatial structure at novel points of observation. Journal of Experimental Psychology: Learning, Memory, and Cognition 15(6), 1157-1165. doi: 10.1037/0278-7393.15.6.1157

Ruggiero, G., Ruotolo, F., \& Iachini, T. (2009). The role of vision in egocentric and allocentric spatial frames of reference. Cognitive Processing, 10(Suppl2), S283-S285. doi:10.1007/s10339-009-0320-9

Ruggiero, G., Ruotolo, F., \& Iachini, T. (2012). Egocentric/allocentric and coordinate/categorical haptic encoding in blind people. Cognitive processing, 13(1), 313-317. doi:10.1007/s10339-012-0504-6

Ruotolo, F., Ruggiero, G., Vinciguerra, M., \& Iachini, T. (2012). Sequential vs simultaneous encoding of spatial information: A comparison between the blind and the sighted. Acta Psychologica, 139(2), 382-389. doi:10.1016/j.actpsy.2011.11.011

Schmidt, S., Tinti, C., Fantino, M., Mammarella, I. C., \& Cornoldi, C. (2013). Spatial representations in blind people: The role of strategies and mobility skills. Acta Psychologica, 142, 43-50. doi:10.1016/j.actpsy.2012.11.010

Sholl, M. J. (2001). The role of a self-reference system in spatial navigation. In D. R. Montello (Ed.), Spatial information theory: Foundations of geographic information science (international conference, COSIT 2001 proceedings), 217-232. Berlin: Springer. 
Szubielska, M. (2014). Strategies for constructing spatial representations used by blind and sighted subjects. Studia Psychologica, 56(4), 273-285. doi:10.1016/j.actpsy.2012.11.010

Taylor, H. A., \& Tversky, B. (1992). Spatial mental models derived from survey and route descriptions. Journal of Memory and Language, 31, 261-292.

Thinus-Blanc, C., \& Gaunet, F. (1997). Representation of space in blind persons: Vision as a spatial sense? Psychological Bulletin, 121(1), 20-42. doi:10.1037/h0080726

Tinti, C., Adenzato, M., Tamietto, M., \& Cornoldi, C. (2006). Visual experience is not necessary for efficient survey spatial cognition: Evidence from blindness. The Quarterly Journal of Experimental Psychology, 59(7), 1306-1328. doi:10.1080/17470210500214275

Wraga, M., Creem, S. H., \& Proffitt, D. R. (2000). Updating displays after imagined object and viewer rotations. Journal of Experimental Psychology: Learning, Memory, and Cognition, 26(1), 151. 


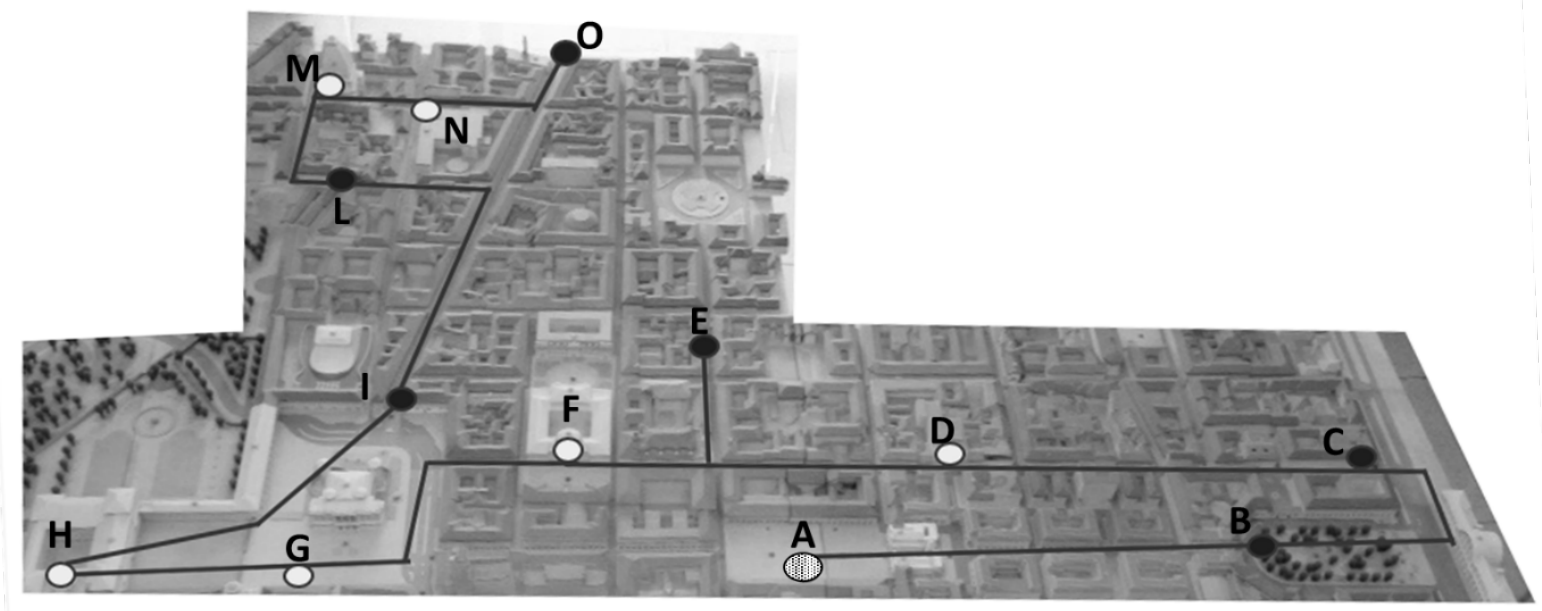

Figure 1. Route of the exploration. On the bottom the starting point of the itinerary, Piazza San Carlo (A); in black, the unknown landmarks; in white, the known landmarks.

List of the landmarks. B: tree with bird's nest (unknown); C: house of Mr. Bianchi, a gentleman with the dog (unknown); D: shopping center "Rinascente” (known); E: house with sundial (unknown); F: Palazzo Carignano (known); G: Palazzo della Regione (known); H: Cathedral of Turin (known); I: location of a movie set (unknown); L: house of Giovanna the cooker (unknown); M: Mole Antonelliana (known); N: RAI (known); O: corner with street artist (unknown). 


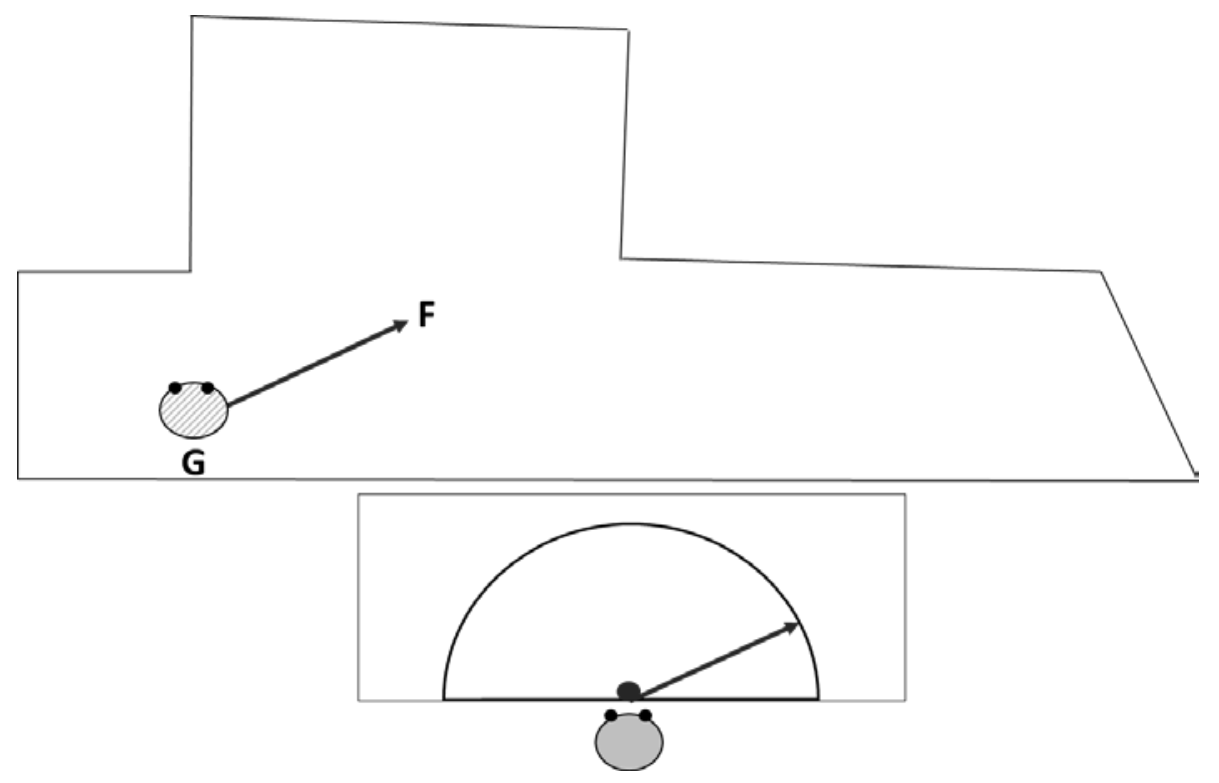

Figure 2. $0^{\circ}$ condition, e.g., "Imagine that you have "Palazzo della Regione” (G) behind of you, point to "Palazzo Carignano" (F)". Above, the position of the map with the perspective imagined by the participant. Below, the tally sheet in front of the participant with the correct location of the pointer. 


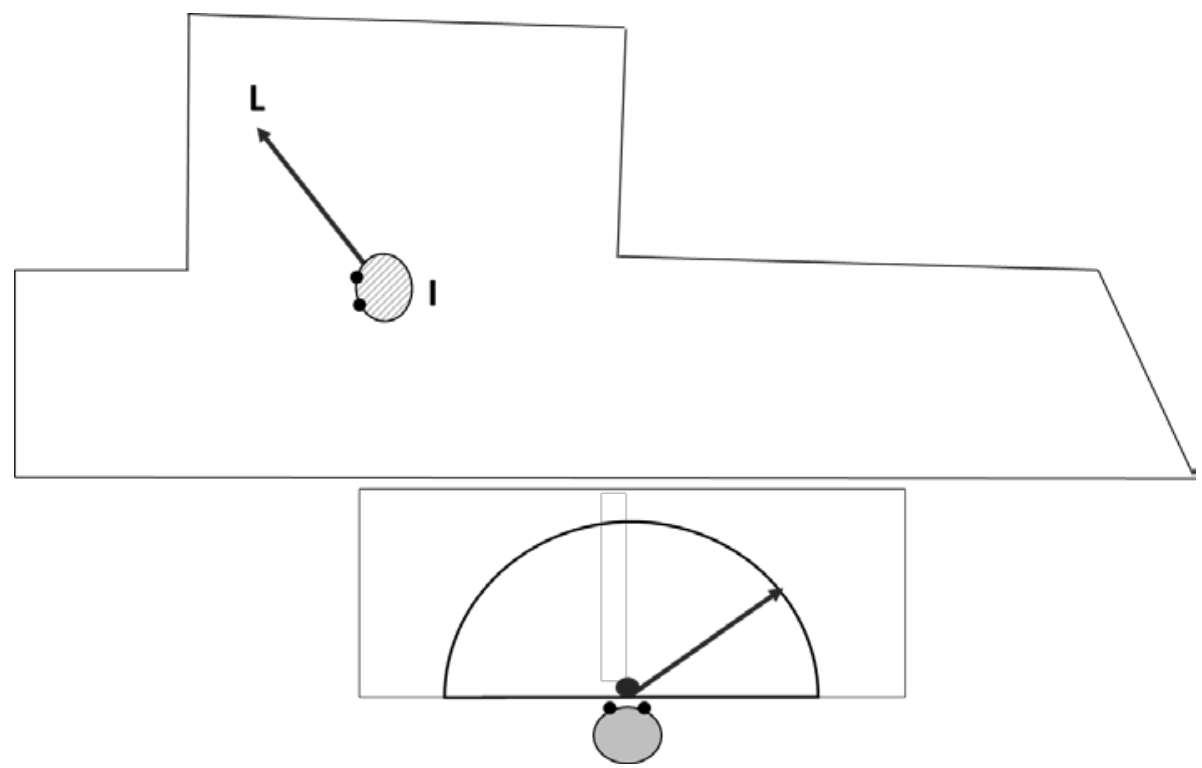

Figure 3. $90^{\circ}$ condition, e.g., "Imagine that you have "the location of the movie set” (I) behind of you, point to "the house of Giovanna, the cooker" (L)". Above, the position of the map with the perspective imagined by the participant. Below, the tally sheet in front of the participant with the correct location of the pointer. 


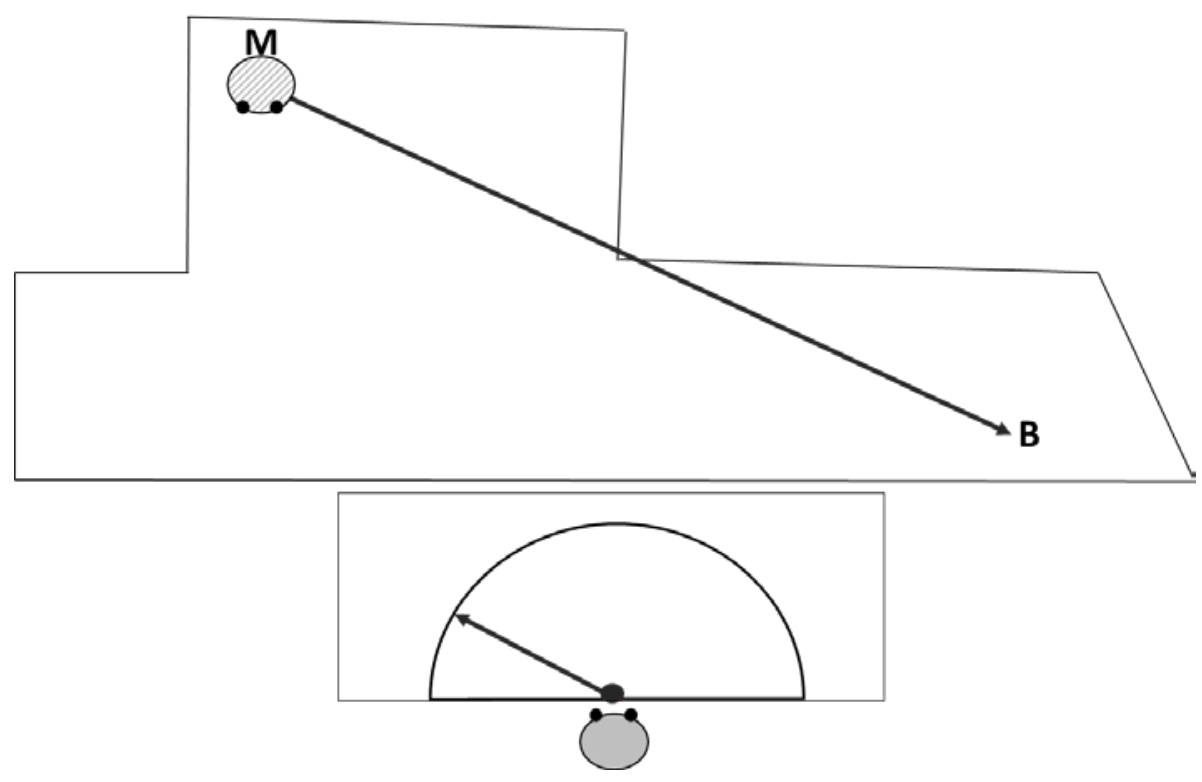

Figure 4. $180^{\circ}$ condition, e.g., "Imagine that you have "the Mole Antonelliana" (M) behind of you, point to "the tree with the bird's nest" (B)". Above, the position of the map with the perspective imagined by the participant. Below, the tally sheet in front of the participant with the correct location of the pointer. 


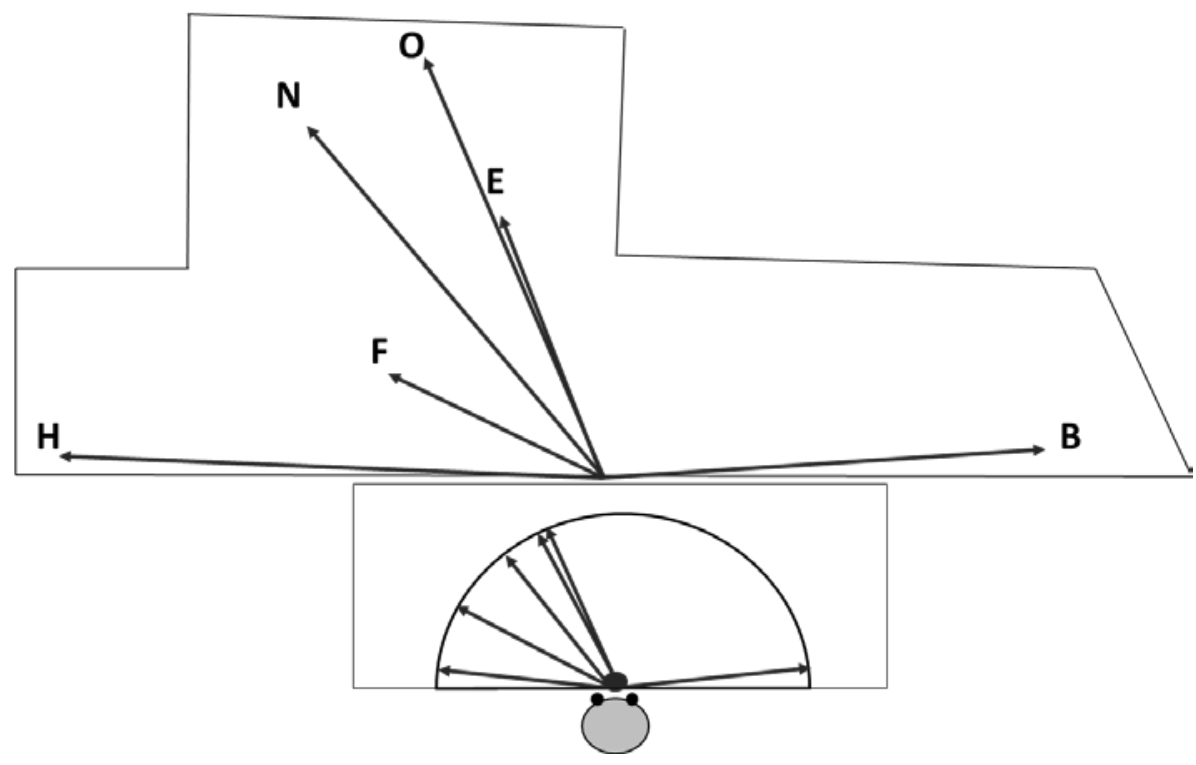

Figure 5. Aligned condition: «Imagine that you have the map in front of you. From this location, point to ...». Above, the position of the imagined map. Below, the tally sheet in front of the participant with the correct locations of the pointer. 


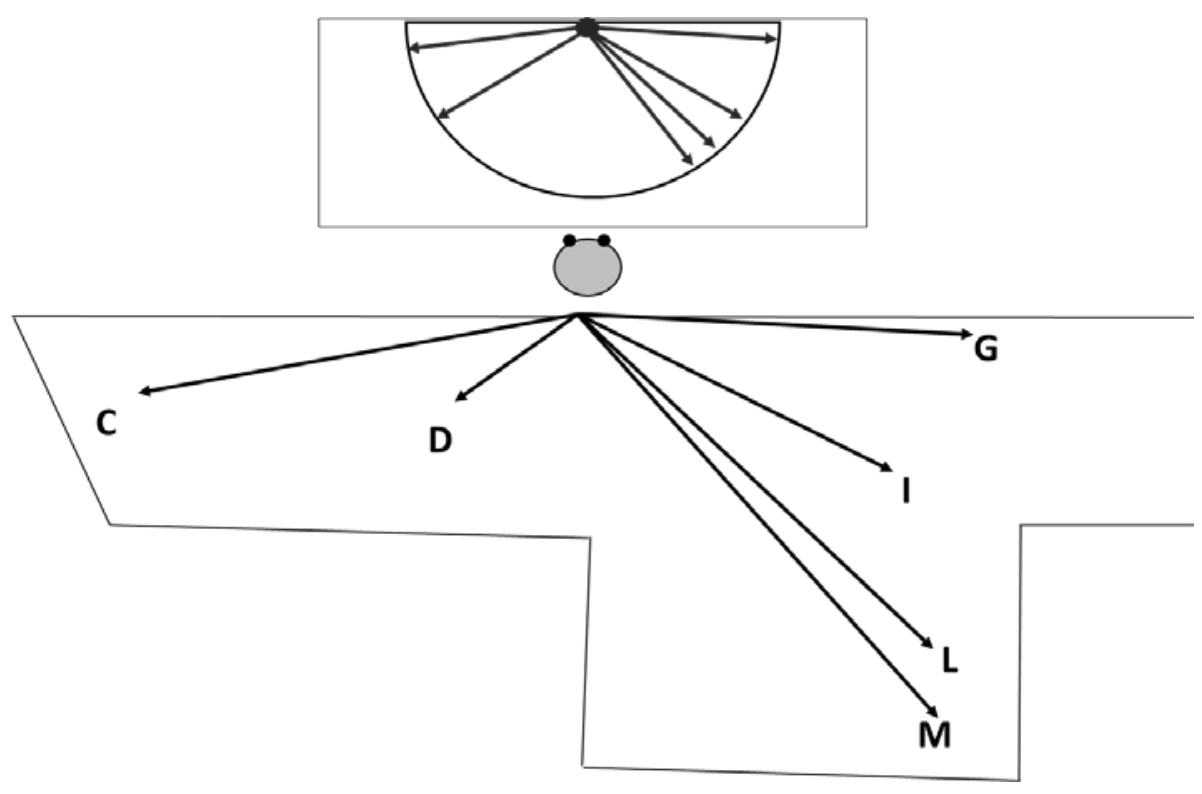

Figure 6. Contra-aligned condition: «Now imagine having the map behind of you. From this position, point to ...». Above, the position of the tally sheet in front of the participant with the correct locations of the pointer. Below, the position of the imagined map. 
Table 1. Adjusted degrees of error in the three conditions of mental rotation by group.

\section{Degrees of mental rotation}

\begin{tabular}{|c|c|c|c|c|c|c|}
\hline \multirow[b]{2}{*}{ Group } & \multicolumn{2}{|c|}{$0^{\circ}$} & \multicolumn{2}{|c|}{$90^{\circ}$} & \multicolumn{2}{|c|}{$180^{\circ}$} \\
\hline & $M$ & $S D$ & $M$ & $S D$ & $M$ & $S D$ \\
\hline Blind & .27 & .13 & .38 & .14 & .45 & .23 \\
\hline Sighted & .22 & .13 & .31 & .16 & .26 & .19 \\
\hline
\end{tabular}


Table 2. Adjusted degrees of error in the two conditions by group.

\begin{tabular}{ccccccc}
\hline & \multicolumn{2}{c}{ Aligned } & & & \multicolumn{2}{c}{ Contra-aligned } \\
\cline { 2 - 3 } \cline { 5 - 6 } Group & $M$ & $S D$ & & $M$ & $S D$ \\
\hline Blind & .16 & .02 & & .27 & .03 \\
Sighted & .13 & .02 & & .17 & .03 \\
\hline
\end{tabular}


Table 3. Frequency of strategies used to solve the tasks in the two groups.

\begin{tabular}{|c|c|c|c|c|c|c|}
\hline & \multicolumn{2}{|c|}{ Survey } & \multicolumn{2}{|c|}{ Route } & \multicolumn{2}{|c|}{ Verbal } \\
\hline & $n$ & $\%$ & $n$ & $\%$ & $N$ & $\%$ \\
\hline Blind & 3 & 15.8 & 12 & 63.2 & 4 & 21.1 \\
\hline Sighted & 9 & 47.4 & 6 & 31.6 & 4 & 21.1 \\
\hline
\end{tabular}


Table 4. Adjusted degrees of error in the three conditions of mental rotation by group and strategy.

Degrees of mental rotation

\begin{tabular}{|c|c|c|c|c|c|c|c|}
\hline \multirow[b]{2}{*}{ Group } & \multirow[b]{2}{*}{ Strategy } & \multicolumn{2}{|c|}{$0^{\circ}$} & \multicolumn{2}{|c|}{$90^{\circ}$} & \multicolumn{2}{|c|}{$180^{\circ}$} \\
\hline & & $M$ & $S D$ & $M$ & $S D$ & $M$ & $S D$ \\
\hline \multirow[t]{3}{*}{ Blind } & Verbal & .25 & .10 & .42 & .11 & .47 & .18 \\
\hline & Survey & .26 & .22 & .31 & .08 & .25 & .24 \\
\hline & Route & .28 & .13 & .39 & .16 & .50 & .22 \\
\hline \multirow[t]{3}{*}{ Sighted } & Verbal & .13 & .03 & .44 & .14 & .43 & .23 \\
\hline & Survey & .20 & .12 & .25 & .10 & .18 & .09 \\
\hline & Route & .29 & .15 & .31 & .22 & .28 & .21 \\
\hline
\end{tabular}


Table 5. Adjusted degrees of error in the two conditions by group and strategy.

\begin{tabular}{|c|c|c|c|c|c|}
\hline \multirow[b]{2}{*}{ Group } & \multirow[b]{2}{*}{ Strategy } & \multicolumn{2}{|c|}{ Aligned } & \multicolumn{2}{|c|}{ Contra-aligned } \\
\hline & & $M$ & $S D$ & $M$ & $S D$ \\
\hline \multirow[t]{3}{*}{ Blind } & Verbal & .21 & .10 & .49 & .16 \\
\hline & Survey & .11 & .02 & .20 & .08 \\
\hline & Route & .16 & .07 & .21 & .08 \\
\hline \multirow[t]{3}{*}{ Sighted } & Verbal & .15 & .09 & .21 & .10 \\
\hline & Survey & .12 & .04 & .14 & .07 \\
\hline & Route & .14 & .08 & .19 & .11 \\
\hline
\end{tabular}

